\title{
Magnetic mapping of iron in rodent spleen
}

Angela R. Blissett广, Brooke Ollander广, Brittany Penn, Dana M. McTigue and Gunjan Agarwal*

List of abbreviations: atomic force microscopy (AFM), immunohistochemistry (IHC), magnetic force microscopy (MFM), optimal cutting temperature (OCT), transmission electron microscopy (TEM), energy dispersive spectroscopy (EDS) 


\begin{abstract}
Evaluation of iron distribution and density in biological tissues is important to understand the pathogenesis of a variety of diseases and the fate of exogenously administered iron-based carriers and contrast agents. Iron distribution in tissues is typically characterized via histochemical (Perl's) stains or immunohistochemistry for ferritin, the major iron storage protein. A more accurate mapping of iron can be achieved via ultrastructural transmission electron microscopy (TEM) based techniques, which involve stringent sample preparation conditions. In this study, we elucidate the capability of magnetic force microscopy (MFM) as a label-free technique to map iron at the nanoscale level in rodent spleen tissue. We complemented and compared our MFM results with those obtained using Perl's staining and TEM. Our results show how MFM mapping corresponded to sizes of iron-rich lysosomes at a resolution comparable to that of TEM. In addition MFM is compatible with tissue sections commonly prepared for routine histology.
\end{abstract}

Keywords: ferritin; iron; lysosomes; magnetic force microscopy; spleen; transmission electron microscopy 


\section{Introduction}

Iron (Fe) is an essential metal involved in a wide spectrum of physiological and pathological processes, e.g. oxygen transport and enzymatic reactions(1). The oxidation state, mineral composition, and confinement of iron play a crucial role in maintaining iron homeostasis. Ferritin is the major iron storage protein found in mammalian tissues, comprising of a nanoscale iron core with up to 4500 iron atoms inside a $~ 12 \mathrm{~nm}$ protein shell. The organs with the largest stores of ferritin (iron) in the human body are the spleen, liver, and bone marrow. In pathological conditions, elevated levels of localized iron deposits can be found at sites of injury(2), in cardiovascular diseases(3), malignancies(4) and neurodegenerative diseases(5). A precise understanding of the quantity and quality of iron distribution in tissues is important for clinical diagnosis and treatment as well as for applications involving delivery of iron-based carriers or contrast agents.

Histological evaluation of ferritin (iron) in biological tissues is commonly accomplished using ferritin-immunohistochemistry (IHC) and/or histochemical iron staining (e.g. Perl's or Turnball's stain). These techniques rely on light microscopy for detection, wherein the observed iron-rich regions are several microns in size. In certain pathologies, a mismatch between ferritin-IHC and histochemical iron staining has been reported(6,7), which could arise due to multiple factors such as the presence of iron-deficient apoferritin or deposition of iron beyond the confinement of a ferritin core(8). Additional factors like antibody type(s) and concentrations, incubation times, and signal enhancement reagents may affect the signal intensity and spatial resolution obtained using $\mathrm{IHC}$ or histochemical stains. 
High-resolution mapping of nanoscale iron deposits in situ can provide a more accurate insight into tissue iron deposits. This can be achieved using ion or electron microprobe based ultrastructural techniques such as secondary ion mass spectroscopy (SIMS)(9) or analytical transmission electron microscopy (TEM)(10). Like the Perl's stain, these approaches are largely based on the chemical composition of the iron particles. However, a major limitation with these ultrastructural techniques is that they require stringent sample preparation protocols not compatible with the rapid and multi-label histological evaluation achievable via light microscopy. The ability to map iron deposits at nanoscale level along with IHC on adjacent sections would be especially advantageous for a more accurate histological evaluation.

One of the unique features of ferritin (iron) deposits which remain un-exploited in histological analysis is the magnetic nature of its iron core. Several in-vitro studies using techniques such as SQUID magnetometry(11), Mossbauer spectroscopy, vibrating sample magnetometry,(12) and nuclear magnetic relaxometry(13) have elucidated the superparamagnetic character of ferritin with an antiferromagnetic sub-lattice arrangement of $\mathrm{Fe}^{3+}$ in its core(14). The superparamagnetic behavior of ferritin above its Neel temperature $\left(>240^{\circ} \mathrm{K}\right)$ arises due to an increase in the number of uncompensated spins resulting from thermal disorder and a linear contribution to its magnetization by an externally applied field $(15,16)$. Magnetic moment of $\mathrm{Fe}^{3+}$ atoms residing in the ferritin core has been determined to be $3.8 \mu_{\mathrm{B}}$ (Bohr magneton)(13), with the entire core possessing a moment of $\sim 200-350$ $\mu_{B}(15,17)$.

Magnetic properties of ferritin have been utilized in magnetometry and relaxometry studies to evaluate the overall iron content ex-vivo in a variety of tissues such as spleen $(18,19)$, 
brain(20), and liver(21). Non-invasive MRI imaging(22) has also been able to track endogenous ferritin iron deposits in-vivo(23). However, these studies fail to provide information on spatial distribution of iron deposits, which is essential to understand iron regulation and function at the subcellular level. Magnetic mapping of tissue sections would be especially advantageous to spatially characterize iron-deposits in situ in histological sections or biopsy samples. It could help resolve a mismatch between ferritin-IHC and histochemical stains as well as that between the iron content of serum ferritin and tissue ferritin in certain pathologies $(24,25)$.

In this study, we investigate the capability of magnetic force microscopy (MFM) to map ferritin (iron) deposits in biological tissue in situ. MFM is a technique utilizing the principles of atomic force microscopy (AFM). It operates by scanning the surface of a sample with a magnetic probe at user defined lift heights to detect the magnetic signature of the scanned sample. While MFM has been extensively used to characterize magnetic domains in solid state devices(26) and magnetic nanoparticles in-vitro(27-29), limited studies exist on applications of MFM for examining tissues or cell samples in-situ(30-33). Previous studies by us(34) and others $(32,35,36)$ have shown how MFM can detect purified ferritin at the single particle level in-vitro. In this study, we examined the rat spleen tissue for ferritin (iron) deposits in-situ using Perl's stain, TEM, and MFM and compared the spatial resolution obtained using these techniques. We demonstrate how MFM can successfully map iron-rich regions in spleen tissue at sub-micron resolution and is compatible with sample preparation typically used for routine histology.

\section{Materials and Methods}




\section{Tissue Collection}

All animal work was performed in agreement with The Ohio State University Institutional Animal Care and Use Committee. Anesthetization of adult male Sprague Dawley rats was accomplished via administration of $80 \mathrm{mg} / \mathrm{kg}$ ketamine/xylazine mixture. Rats $(\mathrm{n}=3)$ were perfused transcardially with distilled water followed by fixation in $250 \mathrm{ml}$ of the primary fixative solution consisting of $4 \%$ paraformaldehyde and $2 \%$ glutaraldehyde in cacodylate buffer (consisting of $0.2 \mathrm{M}$ trihydrate sodium cacodylate at $\mathrm{pH} 7.2$, with $1 \% \mathrm{MgCl}_{2}$ and $0.5 \% \mathrm{CaCl}_{2}$ ). Spleens were then dissected from the animals, cut into $0.5-1 \mathrm{~mm}$ segments and immersionfixed overnight at $4^{\circ} \mathrm{C}$ in primary fixative (as defined above) for TEM processing. Subsequently, tissue was placed in $0.2 \mathrm{M}$ cacodylate buffer for storage before further processing for TEM. For embedding spleen tissue in Optimal Cutting Temperature (OCT) compound, perfusion-fixed tissue (as above) was dissected into $3-5 \mathrm{~mm}$ segments and immersed in OCT filled molds, then snap frozen in liquid nitrogen and stored at $-80^{\circ} \mathrm{C}$ until use.

\section{Transmission Electron Microscopy}

Glutaraldehyde-fixed spleen tissue was processed for TEM analysis, beginning with a 1hour post-stain in 1\% osmium tetroxide (Electron Microscopy Sciences (EMS) Hatfield, PA). Samples underwent a graded ethanol dehydration step (30 - 100\%) followed by an exchange into acetone transition solvent and subsequent infiltration series with a Spurr's resin mixture (EMS \#14300). Samples were polymerized overnight in a $65{ }^{\circ} \mathrm{C}$ oven, after which resin blocks were thick sectioned at $750 \mu \mathrm{m}$ and stained with Methylene Blue-Azure II and Basic Fuchsin 
$\operatorname{stain}(37)$ in order to identify regions of interest (ROI) within spleen samples. Blocks were trimmed down to ROIs, and $40 \mathrm{~nm}$ thin sections were cut on a ultramicrotome (Leica Ultracut UCT, Leica-Microsystems) and collected on 200 mesh copper grids. Sections were examined without further staining on a JEM-1400 TEM (JEOL Ltd. Tokyo, Japan) operating at $80 \mathrm{kV}$ and digital micrographs were captured using a Veleta digital camera (Olympus Soft Imaging Solutions $\mathrm{GmbH}$, Múnster, Germany). For energy dispersive spectroscopy (EDS), the very same grids were imaged in dark field using a Tecnai F20 field emission $200 \mathrm{kV}$ TEM/scanning-TEM (STEM) and X-TWIN lens (FEI) equipped with a HAADF (High-Angle Annular Dark Field) and an EDAX XLT windowless silicon drift detector (SDD).

\author{
Perl's Iron Staining \\ OCT embedded tissue was cut into $10 \mu \mathrm{m}$ thick sections using a HM 505E (Microm) \\ cryostat and mounted onto Superfrost Plus Microscope slides (Fisher Scientific). Sections were \\ rinsed in distilled water 3 times to remove OCT compound, followed by several rinses in $0.1 \mathrm{M}$ \\ phosphate buffer saline (PBS) and 15 minutes in 25\% hydrogen peroxide in methanol. After \\ several more rinses in PBS, tissue was permeabilized for 10 minutes in a $0.1 \%$ Triton $\mathrm{X}-100$ \\ solution in PBS and rinsed thereafter. For staining of ferric iron, a $2 \%$ potassium ferrocyanide \\ solution in $2 \%$ hydrochloric acid solution was incubated on tissue for 30 minutes. All samples \\ were rinsed with $\mathrm{H}_{2} \mathrm{O}$, dehydrated in an ethanol series, cleared with xylene and cover-slipped \\ with Permaslip (Alban Scientific). Slides were imaged using an Axioplan 2 (Zeiss) microscope \\ equipped with an AxioCam color (Zeiss) camera.
}




\section{Magnetic Force Microscopy}

Tissue samples for MFM imaging were prepared using our previously optimized protocol(38). Briefly, the OCT embedded spleen tissue was cryo-sectioned into $10 \mu \mathrm{m}$ thick sections on polylysine coated $0.17-0.25 \mathrm{~mm}$ thick glass coverslips, rinsed three times with ultrapure water, and incubated for 1-hour in ultrapure water to remove residual OCT. Samples were allowed to dry overnight in ambient air. The glass coverslips containing the tissue sections were adhered to a metallic stub and mounted onto the JV scanner of a Multimode AFM equipped with a Nanoscope IIla Controller (Bruker). The tissue sections were visualized using a reflected light module mounted over the AFM head. Iron-rich or iron-poor regions were identified in tissue sections via comparison with the corresponding Perl-stained adjacent sections.

MFM was performed using pre-magnetized high moment (HM) MFM probes (ASYMFMHM, Asylum Research) as described earlier(34). MFM signal resulting from the HM MFM probes was compared with that from standard moment (SM) MFM probes (NSC-18, MikroMasch, Estonia) by using a section of a floppy disc as a test sample (Figure S1 in supporting information). AFM height images (in main mode) and MFM phase images (in lift mode) were recorded on the same region using the interleave scan feature of the Nanoscope software. Images with scan sizes ranging from 5 to $20 \mu \mathrm{m}$ with 512 lines per direction were acquired using a scan speed of 2 to $3 \mathrm{~Hz}$. Several iron-rich or iron-poor regions were scanned at a lift height $(z)$ of $30 \mathrm{~nm}$ to verify the presence vs. absence of MFM signal. For quantitative analysis of MFM signal a subset of iron-rich regions were scanned at lift heights of $0,30,40$ and $50 \mathrm{~nm}$. 
Image analysis

Image J (NIH) was used to analyze the area of iron-rich regions in microscopic images. For analysis of Perl's stained slides, images were subjected to a threshold, to specifically select strongly stained regions. These regions were hand traced and their area ascertained using the particle analysis feature of Image J. Similar hand-tracing analysis was carried out for obtaining area of iron-rich lysosomes in TEM images. No threshold settings were required for TEM images as the boundary of the iron-rich lysosomes could be easily identified in these images. In addition, for TEM images, the intensity for each analyzed lysosomal area was measured by evaluating the average pixel value (0-255) for that area. The MFM phase images were exported as tif files and Image was used to hand-trace and analyze the area of regions showing a negative or a positive phase signal.

The magnitude of MFM phase shifts was ascertained for each region using the section analysis feature of the Nanoscope software. To examine if the phase shift was dependent on various directions across a particle, measurements were repeated along six different directions across a particle (Figure S2, supporting information). The MFM phase shift values were largely independent of the direction of measurement across the particle. For statistical analysis of changes in the area or magnitude of phase shifts regions with lift height, a student's two-tailed paired t-test was performed on data acquired on the same regions at lift heights of 30,40 or 50 nm. A p-value $<0.05$ across two different lift heights was considered significant.

\section{Results}




\section{Perl's stain for iron distribution}

OCT embedded rat spleen was examined for iron distribution by using Perl's iron staining. As shown in Figure $1 \mathrm{~A}$, it is apparent that the distribution of iron in rat spleen tissue was heterogenous with two distinct regions: iron-rich or iron-poor. The areas of intensely stained iron-rich regions were ascertained using image analysis on high magnification images after threshold settings (Figure 1B, C). Frequency distribution of the area of particles above the threshold limit (Figure 1D) showed that the majority of the high-intensity iron-rich regions were in the range of 20 to $40 \mu \mathrm{m}^{2}$ in size. The Perl's stained images were also useful in providing a map of high intensity iron-rich regions for MFM studies.

\section{TEM analysis of iron distribution}

To obtain high-resolution mapping of iron distribution in spleen tissue, bright field TEM imaging and analysis was performed on unstained ultrathin sections. Iron could be identified as electron-dense dark regions in the macrophages present in the red pulp region of the spleen (Figure 2A). Iron was predominantly present in macrophages as nanoscale monodisperse particles in the cytoplasm or as densely packed particles in the lysosomes (Figure 2B), characteristic of the ferritin (iron) core. The iron density in the lysosomes was quantified (in arbitrary 'pixel intensity' units) using image analysis and revealed a non-uniform distribution (Figure $2 \mathrm{C}, \mathrm{D}$ ). The majority ( $75 \%$ ) of the measured lysosomes had a high iron-density (pixel intensity less than the average value of 35 units). Another interesting feature was that a large number of $(\sim 70 \%)$ lysosomes were less than $0.2 \mu \mathrm{m}^{2}$ in size. The high-density iron-rich lysosomes were typically present in this smaller sized lysosome population. 


\section{MFM analysis of iron distribution}

The presence of iron in what was predicted to be iron-poor versus iron-rich regions from Perl's staining was examined using MFM analysis of adjacent unstained sections from OCT embedded tissue. As shown in Figure 4A, no MFM signal could be obtained from iron-poor regions when scanned at lift heights of $30 \mathrm{~nm}$ with the high moment MFM probe. In contrast, when iron-rich regions were subject to MFM analysis, submicron-sized areas exhibiting a mix of negative or positive phase contrast could be observed at a lift height of $30 \mathrm{~nm}$ (Figure 4B, C). These regions were irregular in shape and much larger in size than monodisperse cytoplasmic 
ferritin observed in TEM images. Occasionally, regions with little or no MFM contrast were also observed in iron-rich regions (Figure 4D) indicating a heterogeneous distribution of iron.

To confirm the long-range nature of magnetic interaction, detectable using MFM, a subset of iron-rich regions was scanned at lift heights of $0,30,40$ and $50 \mathrm{~nm}$ (Figure 5). The area of each region exhibiting a negative or positive MFM signal and its corresponding magnitude of phase shift was evaluated for each lift height. It is interesting to note that majority of the regions exhibiting a MFM signal had an area $<0.2 \mu \mathrm{m}^{2}$ in size, consistent with the sizes of iron-rich lysosomes observed in TEM images in Figure 2. Further, the area of positive phase shifts was even smaller than that of negative phase shifts. The area of both the positive and negative phase shift regions decreased with increasing lift heights and a few of these regions failed to show any signal at lift heights of 40 or $50 \mathrm{~nm}$. This decrease in area with lift-height was statistically significant across any two lift-heights considered (Figure 6A) for both positive and negative phase shifts.

Analysis of the magnitude of phase signal revealed a large range $\left(25\right.$ to $\left.165^{\circ}\right)$ for the negative phase shifts whereas the positive phase shift were confined to a narrower range of 100 to $185^{\circ}$ at $z=30 \mathrm{~nm}$. There was no correlation in the magnitude of the phase shift and the area of the region examined. Increasing lift heights resulted in a small but statistically significant decrease in negative phase shift (Figure 6B). Magnitude of positive phase shift showed a decrease in phase signal for a few regions with increasing lift heights, but the results were not statistically significant (Figure 6B). For both negative and positive phase shifts, a few regions observed at $z=30 \mathrm{~nm}$ failed to show any phase signal (indicated by zero area) at higher lift heights. 


\section{Discussion}

In this study, we elucidate the capability of MFM as a histological tool to map the presence of iron deposits in a biological tissue, specifically rodent spleen. We have complemented and compared our MFM results with standard techniques for iron evaluation in histology. Spleen is comprised of two compartments known as red pulp and white pulp. The red pulp filters particles and senescent red blood cells in the blood using the abundant macrophages located in this region, while the white pulp orchestrates immune responses. Iron is primarily located in the ferritin core present in the cytoplasm and in the lysosomes present in macrophages in the red pulp(39-41). Although we did not specifically stain for macrophages or ferritin using IHC, iron could be easily localized using Perl's staining of sections from OCT embedded tissue. Electron dense regions and EDS analysis confirmed the presence of iron in TEM images.

MFM could be performed on identically prepared but unstained sections as that used for Perl's staining. Regions exhibiting MFM signal corresponded to iron-rich regions, while no MFM signal could be observed in iron-poor regions as identified using Perl's staining. A comprehensive analysis of area of the regions exhibiting presence of iron or magnetic signal was performed on Perl's stained, TEM, and MFM images. The area of Perl's stained regions was several microns in size, indicating that histochemical stains can be diffuse and fail to resolve nanoscale dimensions of iron-rich regions present in biological tissues. Ultra-structural techniques, like TEM, provided a more accurate mapping of iron content by characterizing monodisperse cytoplasmic ferritin iron as well as submicron-sized iron-rich lysosomes. 
Although Perl's stained images were used to locate iron-rich regions for MFM imaging, the area of regions displaying a MFM signal matched well with the sizes of iron-rich lysosomes characterized using TEM imaging. These results indicate that MFM could successfully detect iron rich lysosomes in spleen tissue. We thus elucidate that like TEM, MFM can serve as an ultra-structural tool to map iron content at the nanoscale level in biological tissue(s) but merits from minimal sample preparation and a label-free protocol.

A negative phase shift was observed in most regions exhibiting a MFM signal, indicating an attractive force between the probe and the sample. In this study, MFM experiments were performed in the absence of an external magnetic field. The only field experienced by the sample was that emanating from the high-moment MFM probe. Consistent with earlier reports(32,35), we postulate that the MFM probes used in this study induced a magnetic moment in the ferritin (iron) rich lysosomes resulting in a MFM signal. An attractive (negative) phase shift is expected via this mechanism since the direction of induced magnetization for superparamagnetic particles would be parallel to probe magnetization. Interestingly, the magnitude of negative phase shifts (average $\sim 100^{\circ}$ at $z=30 \mathrm{~nm}$ ) in our current study was significantly larger than that reported in earlier MFM studies of ferritin(32,35). We postulate two reasons for this large negative phase shift: (a) use of high moment MFM probes and (b) high ferritin density in lysosomes. As shown in Figure S1, the MFM signal from high moment probes is almost three times larger than the standard moment probes used in these earlier studies. The high-moment probes could even distort the magnetic pattern of a floppy disc. Another factor which could contribute to an enhanced MFM signal, is the resultant magnetization arising from an ensemble of densely packed ferritin in the iron-rich lysosomes. In 
accordance with the Curie law(42), a high ferritin density can result in higher susceptibility values, accounting for a large MFM signal. Such a dependence of MFM signal on the size of ferritin aggregates in-vitro has been reported in previous studies $(35,36)$. The magnitude of negative phase shifts decreased significantly with increasing lift heights, in accordance with the long-range nature of magnetic interaction.

We also observed very strong $\left(>100^{\circ}\right)$ positive (repulsive) phase shifts (at $z=30 \mathrm{~nm}$ ) in smaller-sized regions, which were frequently surrounded by a negative phase shift region. Positive phase shifts have been reported by us(34) and others(36) when MFM studies of purified ferritin were conducted in the presence of an external field $(\sim 0.2 T)$, indicating that the magnetization of ferritin was anti-parallel to that of the probe. The occurrence of repulsive (positive) phase shifts in this study conducted in the absence of an external magnetic field could be explained due to putative ferrimagnetic(43) or diamagnetic(44) domains occurring in lysosomal ferritin or existence of other diamagnetic biological materials. In addition, it is increasingly being recognized that non-magnetic interactions such as electrostatic effects(4547), capacitive coupling(48) and surface topography(49) can also contribute to a positive phase shift in MFM. In our studies, unlike negative phase shift, the strength of positive phase shifts did not decrease significantly with increasing lift heights. These observations along with earlier reports suggest that the origin of positive phase shifts in MFM images could be a combination of magnetic as well as non-magnetic interactions with the MFM probe. Further studies using approaches such as application of electric potentials to the MFM probe(47) or differential MFM imaging(50) may help resolve these contributions. It should also be noted that additional parameters like inter-particle dipole-dipole interactions(51) and heterogeneity in the chemical 
composition and size of the ferritin (iron) cores(52) could also be likely modulators of both negative and positive phase shifts in MFM.

Contradictory to our earlier in-vitro studies on ferritin(34) and superparamagnetic nanoparticles $(27,28)$, we could not observe MFM signal from mono-dispersed ferritin (iron) in biological tissues in-situ. The reasons behind this are not well understood, but we speculate that cytosolic ferritin may be embedded to various depths beyond the range of MFM in the 10 $\mu \mathrm{m}$ thick tissue sections. Ultrathin sectioning may be required to expose these particles to MFM. Our efforts to map iron in ultrathin TEM sections using MFM were unsuccessful (data not shown). This was primarily because the TEM sections were barely visible using the reflected light module on our AFM, hampering our ability to localize iron-rich regions for MFM imaging. Future strategies to enable TEM and MFM imaging on identical samples would need to be devised to overcome these challenges.

In summary our results demonstrate the application of MFM as a label-free tool for histological evaluation of iron content at the nanoscale level. This method could be used for pathologies requiring an accurate mapping of high-density iron-rich areas at the sub-cellular level in a manner compatible with other histological stains. Such an analysis could be especially useful for neurodegenerative diseases, which are characterized by magnetic iron deposits occurring in clumps of 50 to 100 particles(53,54), cardiovascular pathologies(55), as well as diseased spleen which can have significantly higher iron storage(56,57). MFM could also be a useful tool to map the bio-distribution of externally administered magnetic carriers, contrast agents or clustering of ferritin(58) for in-vivo imaging applications. Besides iron quantity, magnetic mapping of iron in physiological vs. pathological conditions could also provide novel 
insights into the quality of iron. Ferritin iron-core is understood to be poly-phasic with at least

three different structures which include ferrihydrite, hematite, and magnetite(59,60). A dysregulation in the relative percentage of these components in pathology could impact the magnetic moment of iron core, which could potentially be discernable using magnetic mapping. Further studies would help establish MFM as a quantitative tool to map the quantity as well as quality of iron deposits in pathological tissues.

\section{Acknowledgements}

We acknowledge Hendrik Colijn of the OSU Center for Microscopy and Analysis (CEMAS) for assistance with EDS studies and Edward P. Calomeni of OSU College of Medicine EM lab for use of the JEOL JEM-1400 TEM. We thank Feng Qin Yin for assistance with animal handling.

\section{References}

1. Abbaspour N, Hurrell R, Kelishadi R. Review on iron and its importance for human health. Vol. 19, Journal of Research in Medical Sciences. 2014. p. 164-74.

2. Sauerbeck A, Schonberg DL, Laws JL, McTigue DM. Systemic iron chelation results in limited functional and histological recovery after traumatic spinal cord injury in rats. Exp Neurol. 2013;248:53-61.

3. Lee FY, Lee TS, Pan CC, Huang AL, Chau LY. Colocalization of iron and ceroid in human atherosclerotic lesions. Atherosclerosis. 1998 Jun;138(2):281-8.

4. Torti S V, Torti FM. Iron and cancer: more ore to be mined. Nat Rev Cancer. 2013;13(5):342-55. 
5. Knovich MA, Storey J a, Coffman LG, Torti S V, Torti FM. Ferritin for the clinician. Blood Rev. 2009;23(3):95-104.

6. van Duijn S, Nabuurs RJA, van Duinen SG, Natté R. Comparison of histological techniques to visualize iron in paraffin-embedded brain tissue of patients with Alzheimer's disease. J Histochem Cytochem. 2013 Nov;61(11):785-92.

7. Morello N, Tonoli E, Logrand F, Fiorito V, Fagoonee S, Turco E, et al. Haemopexin affects iron distribution and ferritin expression in mouse brain. J Cell Mol Med. 2009 Oct;13(10):4192-204.

8. Zecca L, Youdim MBH, Riederer P, Connor JR, Crichton RR. Iron, brain ageing and neurodegenerative disorders. Nat Rev Neurosci. 2004 Nov;5(11):863-73.

9. Quintana C, Bellefqih S, Laval JY, Guerquin-Kern JL, Wu TD, Avila J, et al. Study of the localization of iron, ferritin, and hemosiderin in Alzheimer's disease hippocampus by analytical microscopy at the subcellular level. J Struct Biol. 2006 Jan;153(1):42-54.

10. Collingwood JF, Chong RKK, Kasama T, Cervera-Gontard L, Dunin-Borkowski RE, Perry G, et al. Three-dimensional tomographic imaging and characterization of iron compounds within Alzheimer's plaque core material. J Alzheimers Dis. 2008 Jun;14(2):235-45.

11. Vohralik PF, Lam SKH. NanoSQUID detection of magnetization from ferritin nanoparticles. Supercond Sci Technol. 2009 Jun;22(6):064007.

12. Allen PD, St Pierre TG, Chua-Anusorn W, Ström V, Rao K V. Low-frequency low-field magnetic susceptibility of ferritin and hemosiderin. Biochim Biophys Acta - Mol Basis Dis. 2000;1500:186-96. 
13. Brooks RA, Vymazal J, Goldfarb RB, Bulte JWM, Aisen P. Relaxometry and magnetometry of ferritin. Magn Reson Med. 1998;40(2):227-35.

14. Papaefthymiou GC. The Mössbauer and magnetic properties of ferritin cores. Biochim Biophys Acta. 2010 Aug;1800(8):886-97.

15. Kilcoyne SH, Cywinski R. Ferritin: a model superparamagnet. J Magn Magn Mater. 1995;140-144(PART 2):1466-7.

16. Gilles $\mathrm{C}$, Bonville $\mathrm{P}$, Rakoto $\mathrm{H}$, Broto JM, Wong KKW, Mann S. Magnetic hysteresis and superantiferromagnetism in ferritin nanoparticles. J Magn Magn Mater. 2002;241(23):430-40.

17. Frankel RB, Papaefthymiou GC, Watt GD. Variation of superparamagnetic properties with iron loading in mammalian ferritin. Hyperfine Interact. 1991;66(1-4):71-82.

18. Hackett S, Chua-anusorn W, Pootrakul P, St Pierre TG. The magnetic susceptibilities of iron deposits in thalassaemic spleen tissue. Biochim Biophys Acta - Mol Basis Dis. 2007;1772(3):330-7.

19. Gutiérrez L, Vujić Spasić M, Muckenthaler MU, Lázaro FJ. Quantitative magnetic analysis reveals ferritin-like iron as the most predominant iron-containing species in the murine Hfe-haemochromatosis. Biochim Biophys Acta - Mol Basis Dis. 2012;1822(7):1147-53.

20. Hautot D, Pankhurst Q a, Khan N, Dobson J. Preliminary evaluation of nanoscale biogenic magnetite in Alzheimer's disease brain tissue. Proc Biol Sci. 2003;270 Suppl (Goodman 1953):S62-4.

21. Dubiel SM, Zablotna-Rypien B, Mackey JB. Magnetic properties of human liver and brain 
ferritin. Eur Biophys J. 1999 Jan;28(3):263-7.

22. Mani V, Briley-Saebo KC, Hyafil F, Fayad ZA. Feasibility of in vivo identification of endogenous ferritin with positive contrast MRI in rabbit carotid crush injury using GRASP. Magn Reson Med. 2006 Nov;56(5):1096-106.

23. Gossuin $\mathrm{Y}$, Muller RN, Gillis P. Relaxation induced by ferritin: A better understanding for an improved MRI iron quantification. Vol. 17, NMR in Biomedicine. 2004. p. 427-32.

24. Nielsen $P$, Günther U, Dürken $M$, Fischer R, Düllmann J. Serum ferritin iron in iron overload and liver damage: correlation to body iron stores and diagnostic relevance. J Lab Clin Med. 2000;135(5):413-8.

25. Patel S, Monemian S, Khalid A, Dosik H. Iron Deficiency Anemia in Adult Onset Still's Disease with a Serum Ferritin of 26,387 $\mu \mathrm{g} /$ L. Anemia. 2011;2011:184748.

26. Savla M, Pandian RP, Kuppusamy P, Agarwal G. Magnetic Force Microscopy of an Oxygen-Sensing Spin-Probe. Isr J Chem. 2008;48:33-8.

27. Schreiber S, Savla M, Pelekhov D V, Iscru DF, Selcu C, Hammel PC, et al. Magnetic force microscopy of superparamagnetic nanoparticles. Small. 2008 Feb;4(2):270-8.

28. Nocera TM, Chen J, Murray CB, Agarwal G. Magnetic anisotropy considerations in magnetic force microscopy studies of single superparamagnetic nanoparticles. Nanotechnology. 2012;23(49):495704.

29. Suzuki H, Tanaka T, Sasaki T, Nakamura N. High-Resolution Magnetic Force Microscope Images of a Magnetic Particle Chain Extracted from Magnetic Bacteria AMB-1. Jpn J Appl Phys. 1998;37(11):1343-5. 
30. Proksch R, Schaffer T. Magnetic force microscopy of the submicron magnetic assembly in a magnetotactic bacterium. Appl Phys Lett. 1995;66(19):2582-4.

31. Shen H, Long D, Zhu L, Li X-Y, Dong Y, Jia N, et al. Magnetic force microscopy analysis of apoptosis of HL-60 cells induced by complex of antisense oligonucleotides and magnetic nanoparticles. Biophys Chem. 2006;122:1-4.

32. Passeri D, Dong C, Reggente M, Angeloni L, Barteri M, Scaramuzzo FA, et al. Magnetic force microscopy: quantitative issues in biomaterials. Biomatter. 2014 Jan;4(1):e29507.

33. Dunn JR, Fuller M, Zoeger J, Dobson J, Heller F, Hammann J, et al. Magnetic material in the human hippocampus. Brain Res Bull. 1995;36(2):149-53.

34. Nocera TM, Zeng Y, Agarwal G. Distinguishing ferritin from apoferritin using magnetic force microscopy. Nanotechnology. 2014 Nov 21;25(46):461001.

35. Hsieh S, Hsieh CW, Zheng B. Ferritin protein imaging and detection by magnetic force microscopy. Chem Commun. 2010;46(10):1655-7.

36. Martinez R V., Chiesa M, Garcia R. Nanopatterning of Ferritin Molecules and the Controlled Size Reduction of Their Magnetic Cores. Small. 2011;7:2914-20.

37. Humphrey CD, Pittman FE. A simple methylene blue-azure II-basic fuchsin stain for epoxy-embedded tissue sections. Stain Technol. 1974;49:9-14.

38. Tonniges JR, Albert B, Calomeni EP, Roy S, Lee J, Mo X, et al. Collagen Fibril Ultrastructure in Mice Lacking Discoidin Domain Receptor 1. Microsc Microanal. 2016 Jun;22(3):599_ 611.

39. Britten $\mathrm{KJ}$, Jones $\mathrm{DB}$, De Sousa M, Wright $\mathrm{DH}$. The distribution of iron and iron binding 
proteins in spleen with reference to Hodgkin's disease. Br J Cancer. 1986 Aug;54(2):27786.

40. Gutiérrez L, Quintana C, Patiño C, Bueno J, Coppin H, Roth MP, et al. Iron speciation study in Hfe knockout mice tissues: magnetic and ultrastructural characterisation. Biochim Biophys Acta. 2009 Jun;1792(6):541-7.

41. Tamion A, Hillenkamp M, Hillion A, Maraloiu VA, Vlaicu ID, Stefan M, Ghica D, Rositi R, Chauveau F, Blanchin MG WM and D V. Ferritin surplus in mouse spleen 14 months after intravenous injection of iron oxide nanoparticles at clinical dose. Nano Res. 2016;9(8):2398-410.

42. Lázaro FJ, Gutiérrez L, Abadía AR, Romero MS, López A. Biological tissue magnetism in the frame of iron overload diseases. J Magn Magn Mater. 2007;316(2 SPEC. ISS.):126-31.

43. Michel FM, Barrón V, Torrent J, Morales MP, Serna CJ, Boily J-F, et al. Ordered ferrimagnetic form of ferrihydrite reveals links among structure, composition, and magnetism. Proc Natl Acad Sci U S A. 2010;107(7):2787-92.

44. Pankowska M, Dobek A. Linear and nonlinear magneto-optics of ferritin. J Chem Phys. 2009;131(1):015105.

45. Angeloni L, Passeri D, Reggente M, Mantovani D, Rossi M. Removal of electrostatic artifacts in magnetic force microscopy by controlled magnetization of the tip: Application to superparamagnetic nanoparticles. Sci Rep. 2016;6(April):1-14.

46. Schwarz A, Wiesendanger R. Magnetic sensitive force microscopy. Vol. 3, Nano Today. 2008. p. 28-39. 
47. Jaafar M, Iglesias-Freire O, Serrano-Ramón L, Ibarra MR, de Teresa JM, Asenjo A.

Distinguishing magnetic and electrostatic interactions by a Kelvin probe force microscopy-magnetic force microscopy combination. Beilstein J Nanotechnol. 2011;2(c):552-60.

48. Li LH, Chen Y. Electric contributions to magnetic force microscopy response from graphene and MoS2 nanosheets. J Appl Phys. 2014;116(21):213904.

49. Angeloni L, Passeri D, Reggente M, Rossi M, Mantovani D, Lazzaro L, et al. Experimental issues in magnetic force microscopy of nanoparticles. In: AIP Conference Proceedings. 2015.

50. Wang Y, Wang Z, Liu J HL. Differential magnetic force microscope imaging. Scanning. $2015 ; 37(2): 112-5$.

51. Levy M, Wilhelm C, Luciani N, Deveaux V, Gendron F, Luciani A, et al. Nanomagnetism reveals the intracellular clustering of iron oxide nanoparticles in the organism. Nanoscale. 2011 Oct 5;3(10):4402.

52. López A, Gutiérrez L, Lázaro FJ. The role of dipolar interaction in the quantitative determination of particulate magnetic carriers in biological tissues. Phys Med Biol. 2007 Aug 21;52(16):5043-56.

53. Ward RJ, Zucca FA, Duyn JH, Crichton RR, Zecca L. The role of iron in brain ageing and neurodegenerative disorders. Lancet Neurol. 2014 Oct;13(10):1045-60.

54. Kirschvink JL, Kobayashi-Kirschvink A, Woodford BJ. Magnetite biomineralization in the human brain. Proc Natl Acad Sci U S A. 1992 Aug 15;89(16):7683-7. 
55. Whitnall M, Suryo Rahmanto Y, Huang ML-H, Saletta F, Lok HC, Gutiérrez L, et al. Identification of nonferritin mitochondrial iron deposits in a mouse model of Friedreich ataxia. Proc Natl Acad Sci U S A. 2012 Dec 11;109(50):20590-5.

56. Morgan EH, Walters MN. Iron storage in human disease: Fractionation of hepatic and splenic iron into ferritin and haemosiderin with histochemical correlations. J Clin Pathol. 1963 Mar;16(2):101-7.

57. Kopáni M, Miglierini M, Lančok A, Dekan J, Čaplovicová M, Jakubovský J, et al. Iron oxides in human spleen. Biometals. 2015 Oct;28(5):913-28.

58. Gossuin Y, Gillis P, Hocq A, Vuong QL, Roch A. Magnetic resonance relaxation properties of superparamagnetic particles. Wiley Interdiscip Rev Nanomed Nanobiotechnol. Jan;1(3):299-310.

59. Cowley JM, Janney DE, Gerkin RC, Buseck PR. The structure of ferritin cores determined by electron nanodiffraction. J Struct Biol. 2000 Sep;131(3):210-6.

60. Gálvez N, Fernández B, Sánchez P, Cuesta R, Ceolín M, Clemente-León M, et al. Comparative structural and chemical studies of ferritin cores with gradual removal of their iron contents. J Am Chem Soc. 2008 Jun 25;130(25):8062-8. 


\section{Figure Legends}

Figure 1: Perl's staining for iron in OCT embedded rat spleen sections. Low magnification image (panel A) indicates overall iron distribution within the spleen. Iron-rich (blue/black) vs. ironpoor (unstained) regions can be easily identified in these images. Higher magnification images (panel B), were set to a global threshold (panel C) and the area of the highly-stained regions was evaluated (histogram in D).

Figure 2: TEM examination of unstained sections of rat spleen shows that iron is localized in electron dense regions (arrows) in the macrophages present in the red pulp (A). High magnification TEM images reveal that nanoscale iron particles (resembling ferritin cores) are dispersed in the cytoplasm and are also present in high densities in the lysosomes (B). The density of iron in lysosomes is variable with the darkest lysosomes being highly "iron rich" (C). Analysis of lysosomal area and pixel intensity indicates that majority of the lysosomes are $<0.2$ $\mu \mathrm{m}^{2}$ in area with high iron density (low pixel intensity) (D).

Figure 3: Scanning transmission electron microscopy (STEM) image of a TEM section (A). Iron particles are visible as nanoscale bright dots. Energy-dispersive X-ray spectroscopy (EDS) spectrum on scanned regions ( $B$ and $C$ ) shows the presence of iron peaks in the cytoplasm (B) and at a higher intensity in the lysosomes (C).

Figure 4: Topographic and phase images obtained from iron-poor and iron-rich regions (as indicated) on unstained OCT embedded spleen sections using a high moment MFM probe. Iron- 
rich regions were identified using Perl's staining of adjacent sections as shown in Figure 1. Ironrich regions frequently showed a MFM signal at a lift height of $30 \mathrm{~nm}$ whereas iron-poor regions did not. Regions with MFM signal revealed a mix of negative (dark) and positive (bright) phase shifts.

Figure 5: MFM phase images from an iron-rich region of the spleen tissue acquired at four different lift heights (as indicated). The height image of the corresponding region is shown on the top right. Quantitative analysis of the magnitude of MFM phase shift and area of regions with negative (blue) or positive (red) phases at the various lift heights is shown. A few regions with a MFM signal at $\mathrm{z}=30 \mathrm{~nm}$ were not visible (indicated by zero area) at higher lift heights.

Figure 6: Average values of (A) area and (B) phase of regions exhibiting a MFM signal with positive ( $n=12$, red) or negative $(n=19$, blue) phase shifts as shown in Figure 5. Area of all regions exhibiting either a positive or a negative phase shift showed a statistically significant decrease with increasing lift heights $\left({ }^{*} p<0.05 ;{ }^{*} p<0.01\right)$. There was a statistically significant decrease in the magnitude of negative phase with lift heights. Although the magnitude of positive phase shift decreased with increasing lift heights for some regions, the results were not statistically significant $(p>0.10)$. 
A
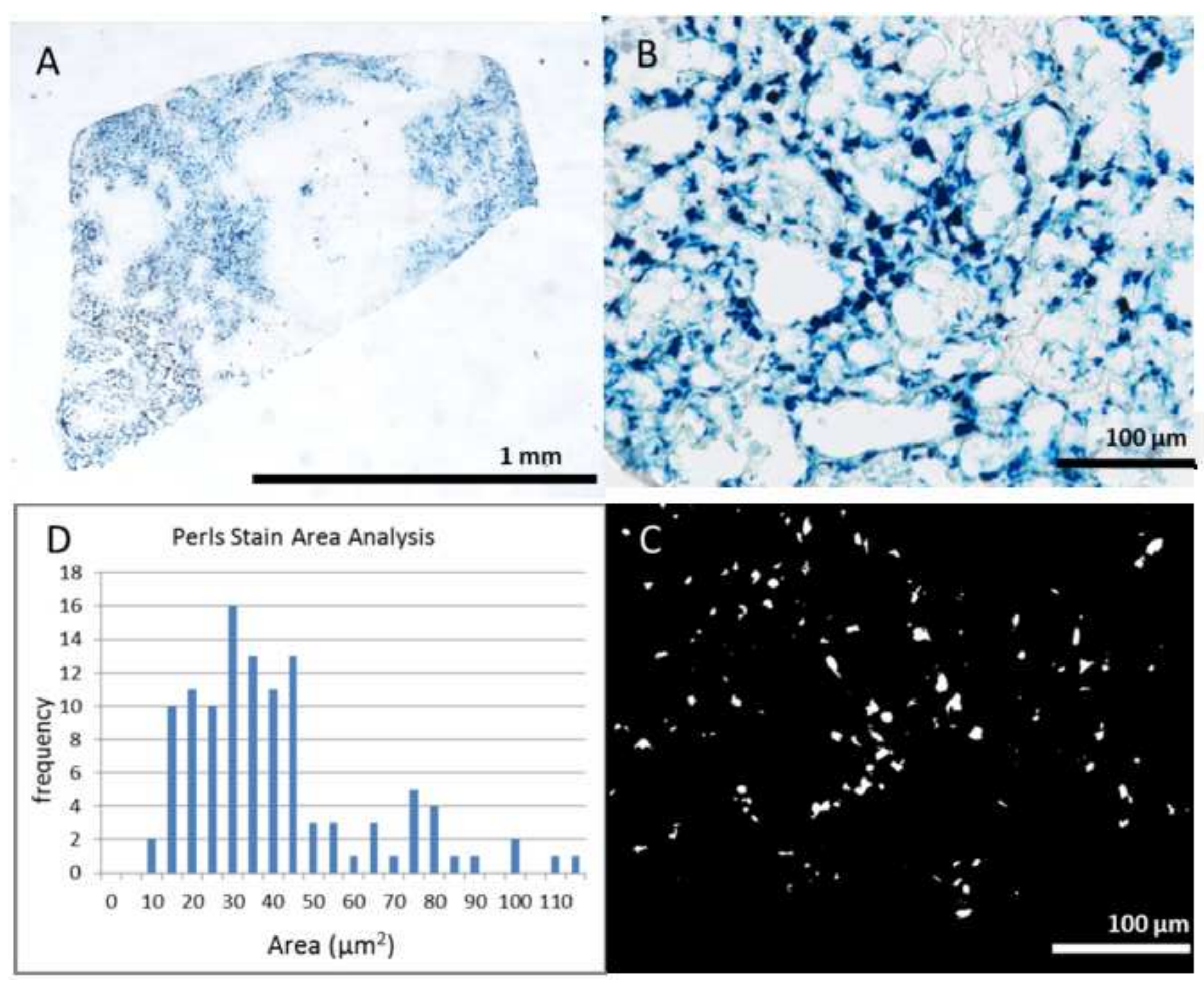

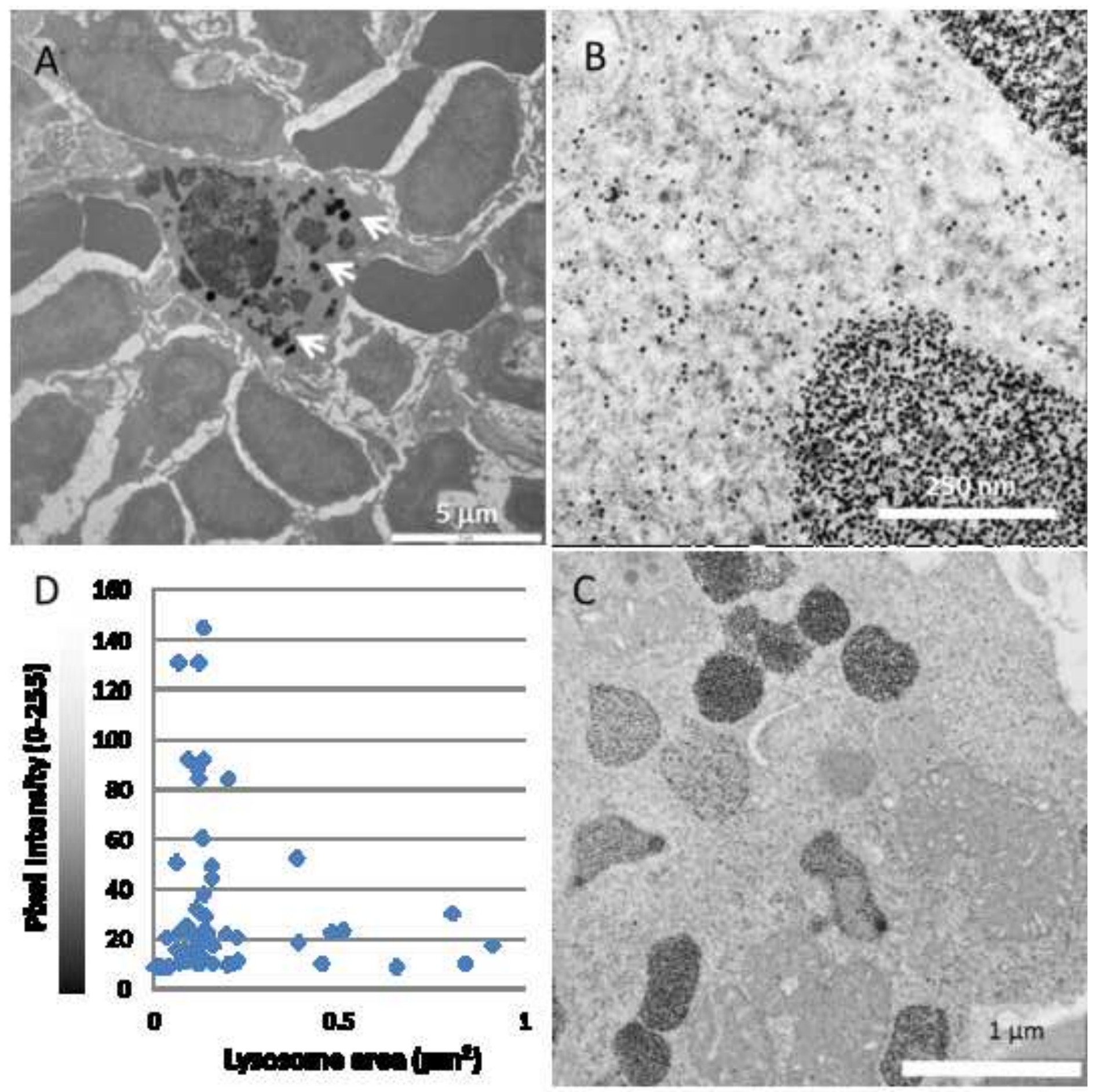

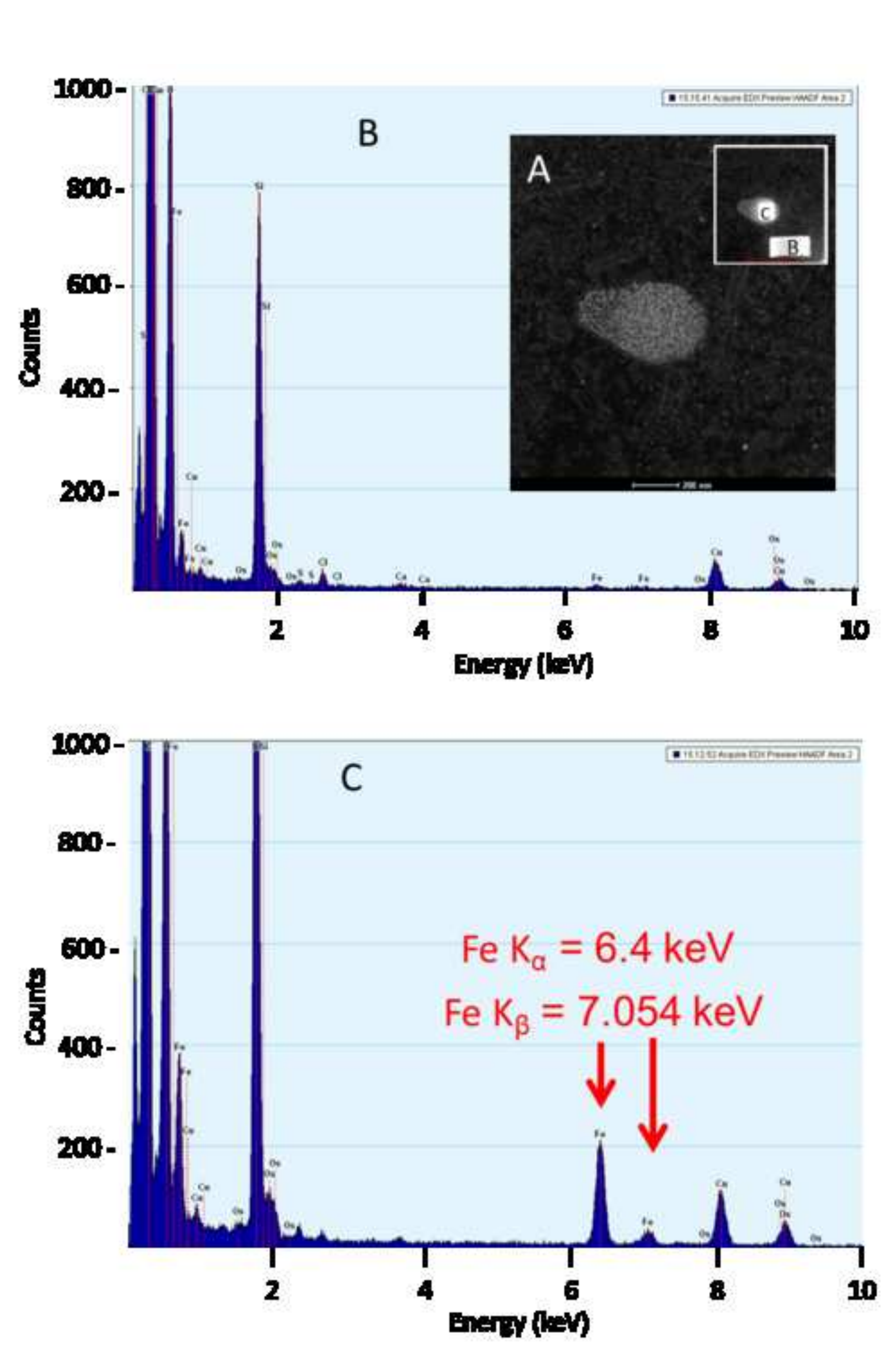

Figure3

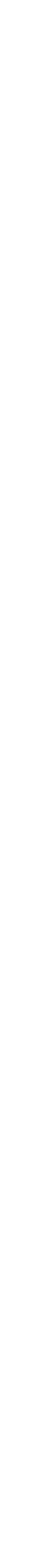

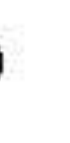
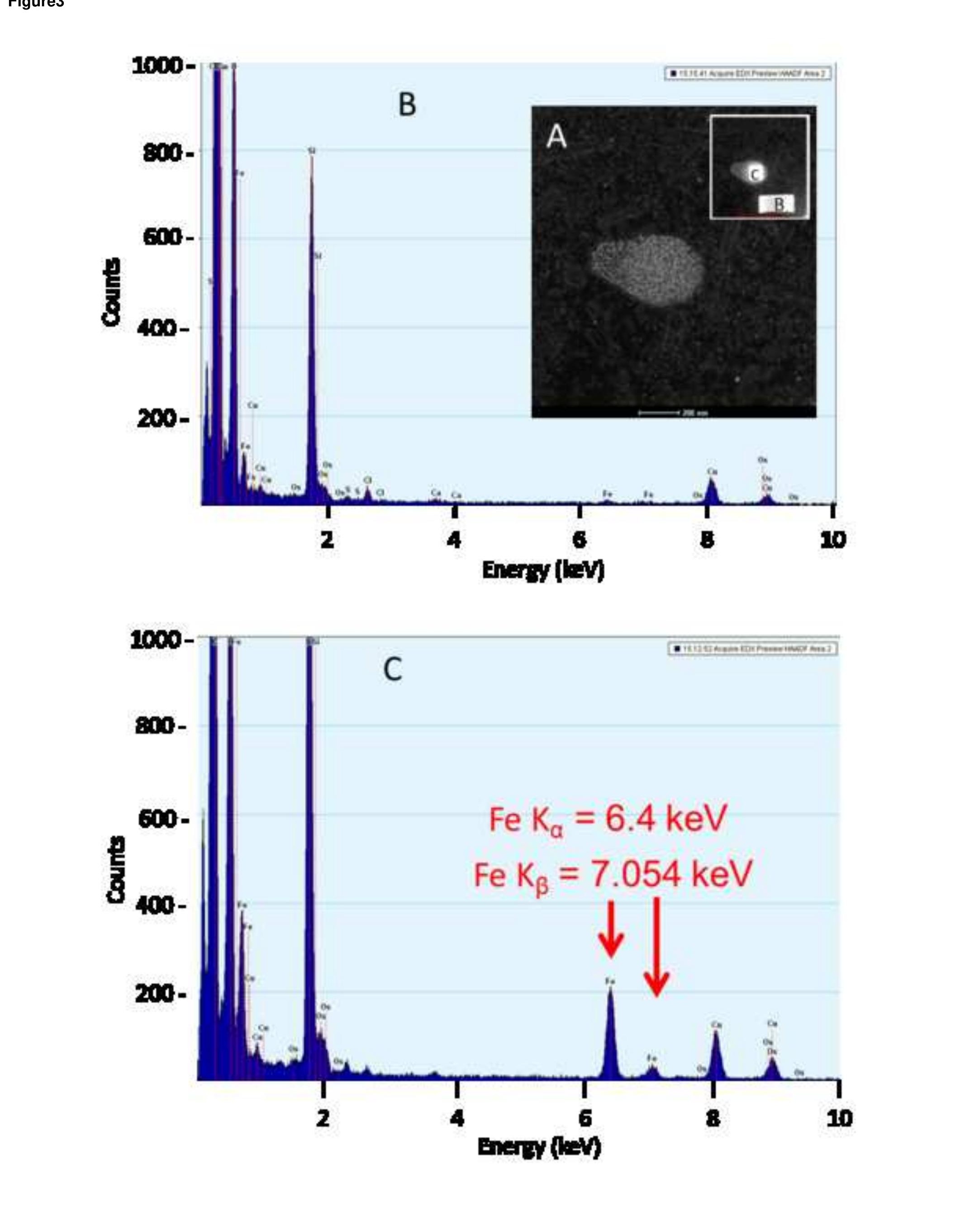

Enter (1) 

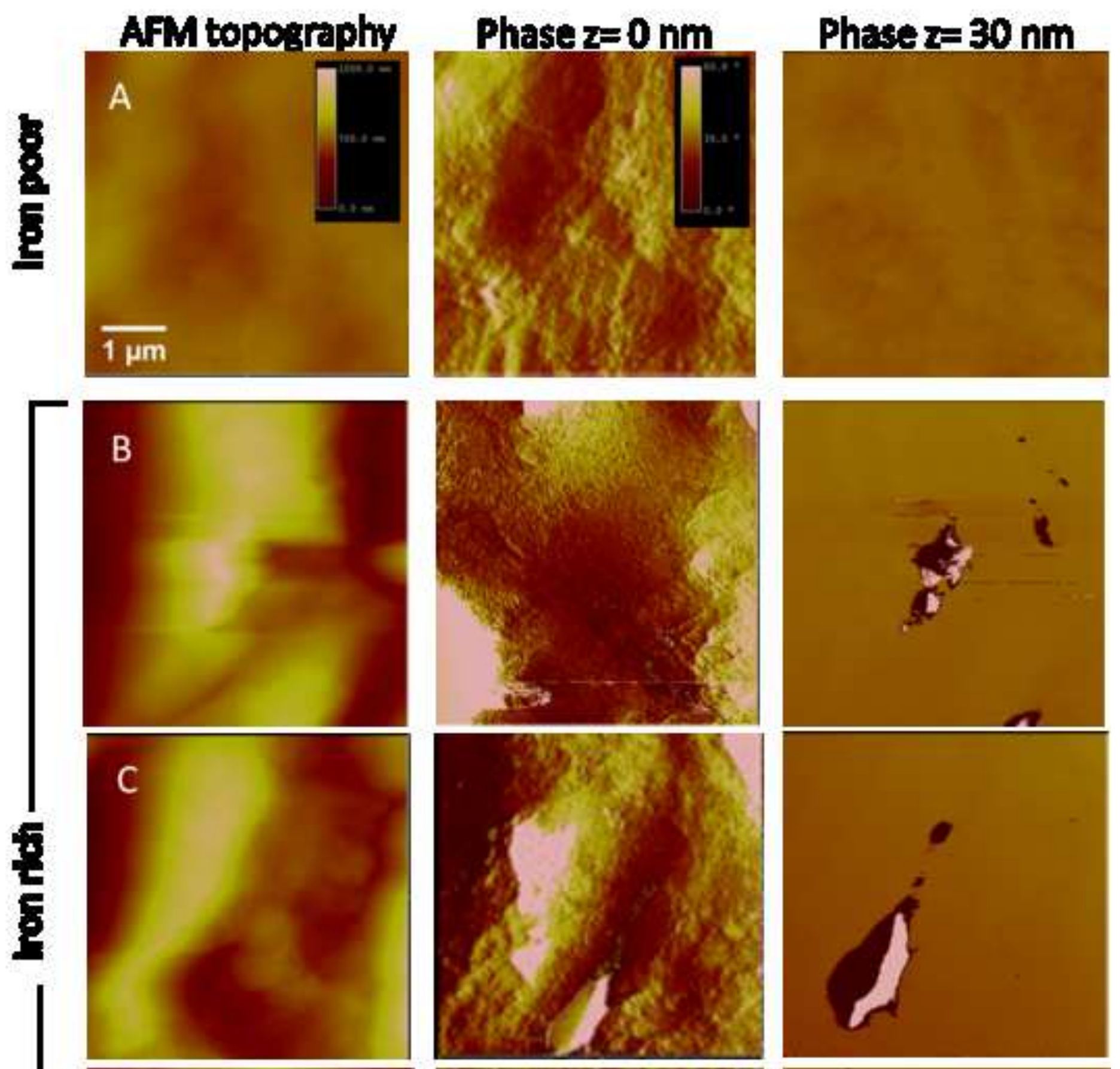

D
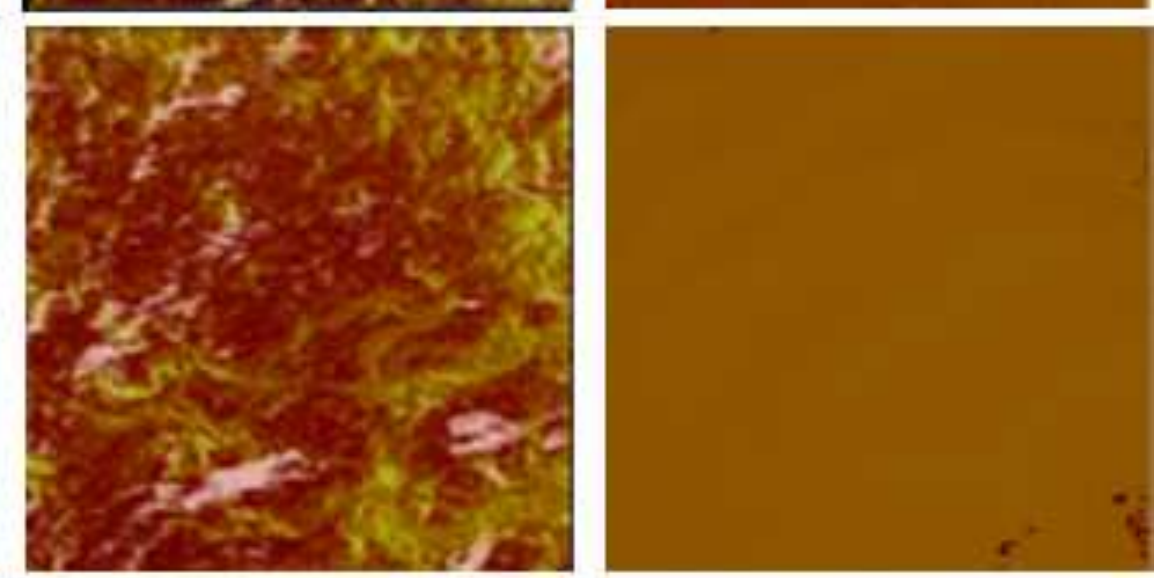

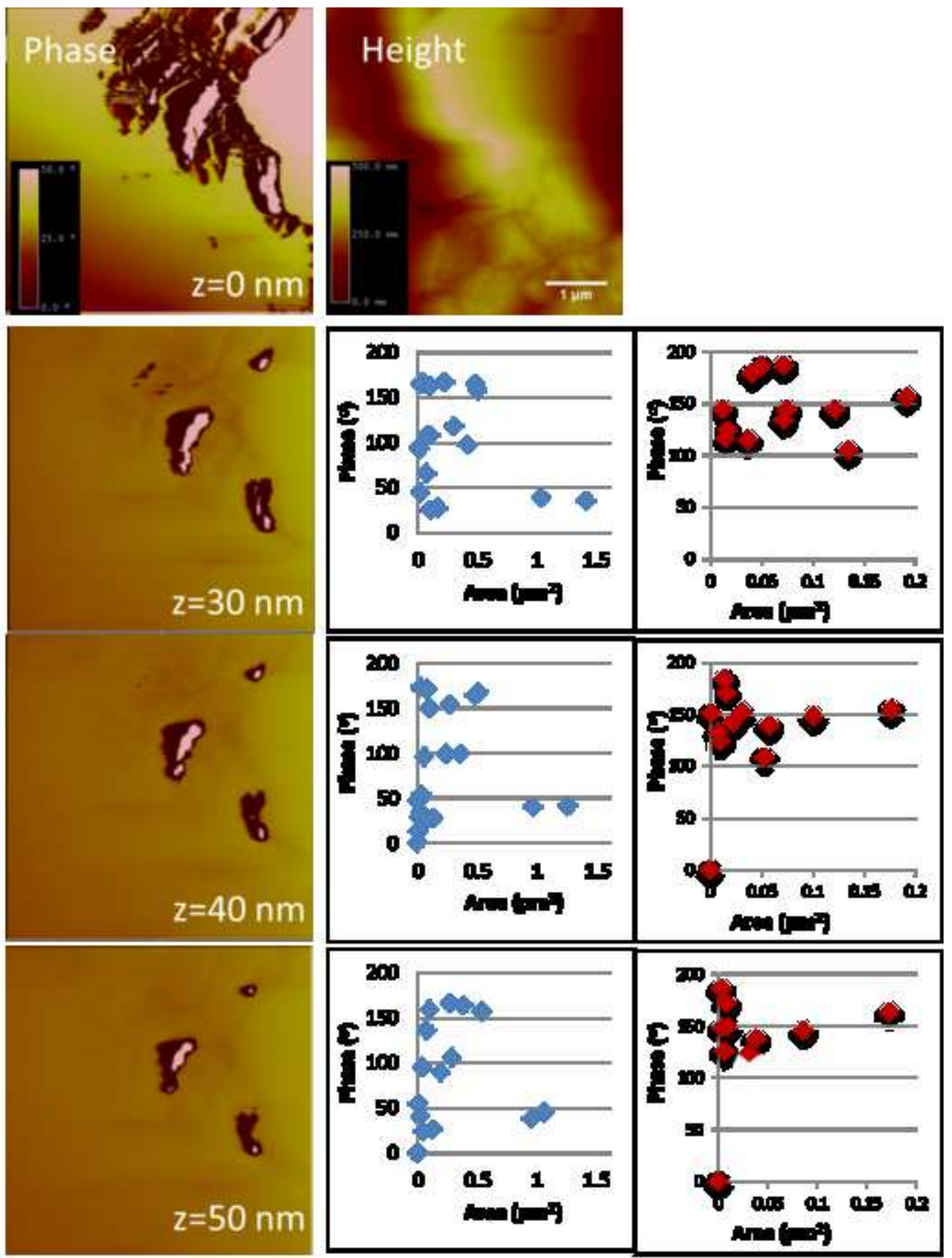

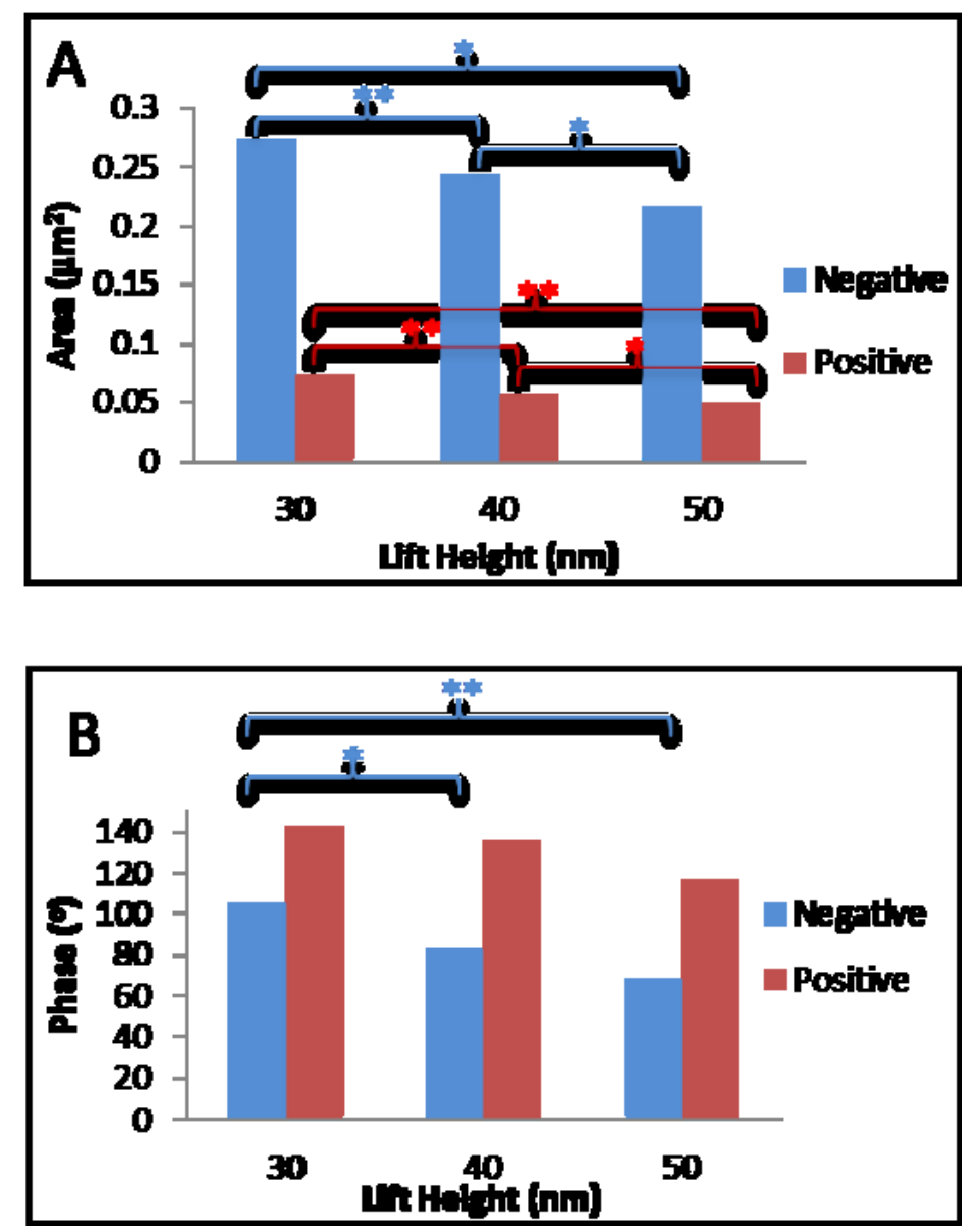

Negrtue

Positive 

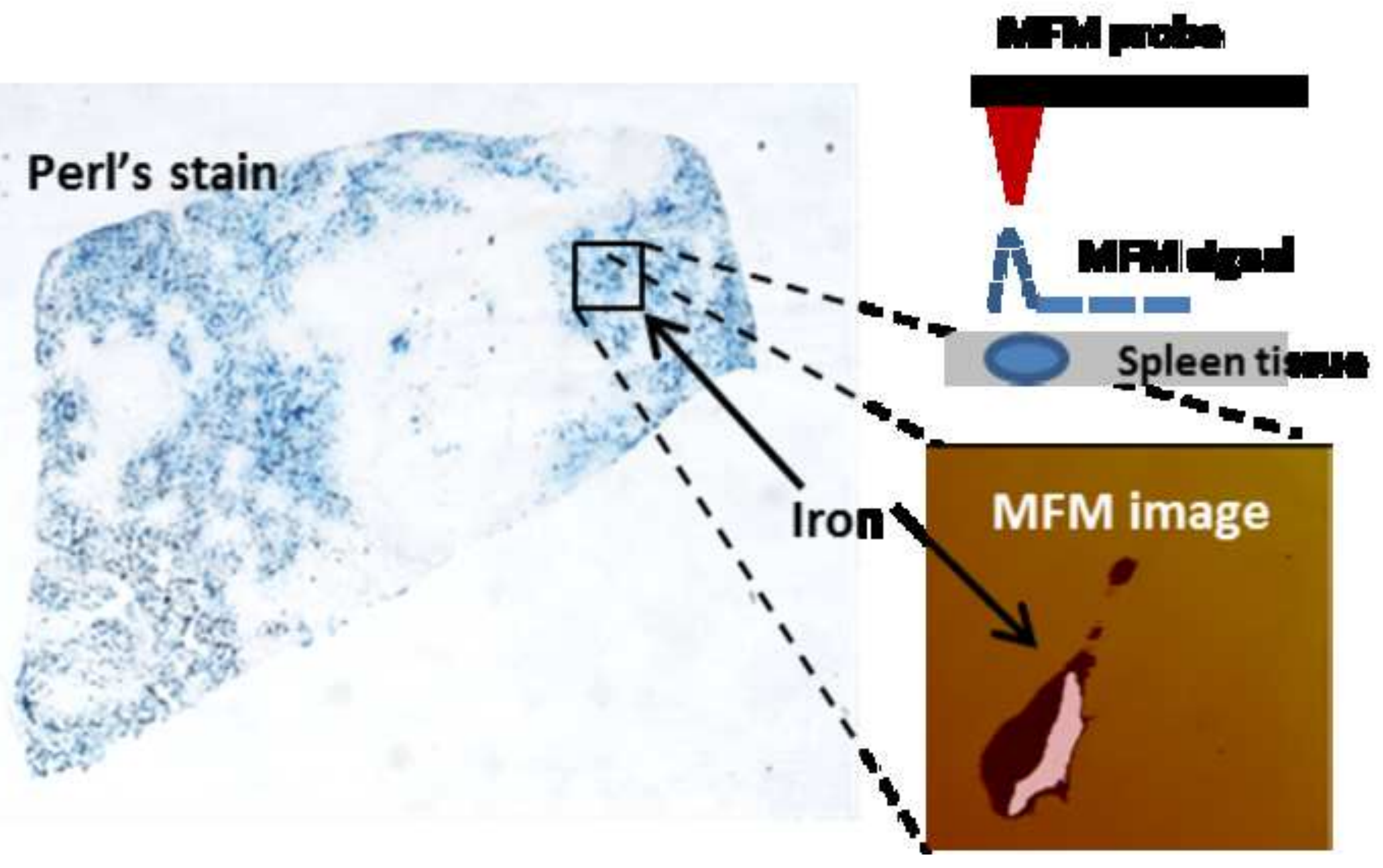